\title{
Neural Networks and Particle Swarm Optimization for Function Approximation in Tri-SWACH Hull Design
}

\author{
Sam Palmer \\ Dept. of Computer Science \\ University College London \\ London WC1E 6BT \\ +44 (0)207679 7039 \\ ucabsdp@ucl.ac.uk
}

\author{
Denise Gorse \\ Dept. of Computer Science \\ University College London \\ London WC1E 6BT \\ +44 (0)207679 7214 \\ ucacdgo@ucl.ac.uk
}

\author{
Ema Muk-Pavic \\ Dept. of Mechanical Engineering \\ University College London \\ London WC1E 6BT \\ +44 (0)207679 3252 \\ e.muk-pavic@ucl.ac.uk
}

\begin{abstract}
Tri-SWACH is a novel multihull ship design that is well suited to a wide range of industrial, commercial, and military applications, but which because of its novelty has few experimental studies on which to base further development work. Using a new form of particle swarm optimization that incorporates a strong element of stochastic search, Breeding PSO, it is shown it is possible to use multilayer nets to predict resistance functions for Tri-SWACH hullforms, including one function, the Residual Resistance Coefficient, which was found intractable with previously explored neural network training methods.
\end{abstract}

\section{Categories and Subject Descriptors}

- Computing methodologies Bio-inspired approaches; Particle swarm optimization; Applied computing Engineering; Hullform resistance prediction;

\section{Keywords}

Particle swarm optimization; function approximation; TriSWACH; multihull resistance; hullform design.

\section{INTRODUCTION}

As the world's needs for natural resources increase, technology develops to satisfy the demand. The oceans are a resource that has so far not been extensively tapped; however as demands grow and onshore resources are increasingly depleted we are turning toward this potentially massive resource. In addition to the traditional fishing, shipping and offshore oil and gas industries we now see progress in food production, seabed mining, and tidal and offshore wind energy harvesting. All these sectors require the development of technologies able to deal with working in the ocean environment, the main challenges being harsh climatic conditions, remoteness, communication and security issues, and environmental impact.

There are currently a large number of creative ideas in ship design fast-tracking toward prototypes able to address some of these new challenges, Trimaran Small Waterplane Area Centre Hull (TriSWACH), being one of them. However due to the novelty of the design there is a lack of experimental data, and in addition no analytical solution or reliable numerical method able to approximate water resistance for this hullform.

Artificial neural networks (ANNs) and other bio-inspired metaheuristic techniques such as particle swarm optimization offer the possibility of being able to extract the underlying physics from limited experimental data, to build a resistance model that could be used to refine and scale up the Tri-SWACH hull design. In the current work it will be shown that a multilayer perceptron, training with Breeding PSO (BrPSO), a new form of particle swarm optimization developed for this work, can be used to predict Tri-SWACH hull resistance.

\section{BACKGROUND}

\subsection{Tri-SWACH}

Tri-SWACH is a novel ship hullform concept presenting a hybrid of a SWATH (Small Waterplane Area Twin Hull) centerhull and two small sidehulls, as depicted in outline in Figures 1 and 2. Initial research shows that this vessel has outstanding motion performance allowing for operations in very harsh weather conditions.

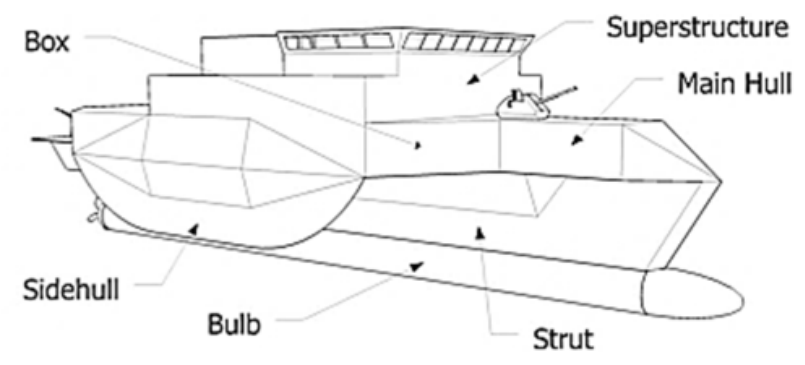

Figure 1. Tri-SWACH hullform (figure from [3]).

\subsection{Previous Application of ANNs to Tri- SWACH Design}

While neural networks and other bio-inspired metaheuristics have found many applications in engineering and design, these methods have more slowly infiltrated the field of naval architecture, though these applications have been notably successful and are growing in number [1]. Of particular interest are cases in which ANNs have been used as prediction tools for multihull resistance. Multihulls pose particular problems for designers due to the complex wave interactions created by the hulls; these wave patterns, which are very difficult to model, depend on the sidehull configuration and can either cause a destructive (decreasing resistance) or constructive (increasing resistance) effect. ANNs however have been shown to be able to successfully predict both 
catamaran [2] and trimaran [3] resistance, outperforming traditional statistical regression models, which gives confidence that similar methods could be applied also to Tri-SWACH.

Two prior studies $[4,5]$ have been carried out with direct bearing on the current work. The results of Carter [4] were very encouraging, showing it was possible to train an ANN to capture the overall shape of the Total Resistance $\left(\mathrm{R}_{\mathrm{T}}\right)$ function shown in Figure 4. However it proved difficult to predict the shape of the $\mathrm{R}_{\mathrm{T}}$ curve in detail, in particular the 'hump' in the resistance curve due to wave interference effects, whose shape and position as a function of speed depend on the sidehull configuration. The work of Chaggar [5] represented a significant advance, as in this case it proved possible to predict the full shape of the $\mathrm{R}_{\mathrm{T}}$ curve, including the prismatic hump. The major differences between this work and [4] were in the learning methodology (Bayesian Regularization in [5], the Levenberg-Marquardt method in [4]) and in the selection of data (data from a single source, the Webb Institute [6], was used in [5], avoiding possible problems introduced by data gathered in different locations).

However this later study too had its limitations. It was originally intended to predict resistance solely on the basis of the sidehull geometry and a single speed-related input, Froude number $\left(F_{r}\right)$, but it proved impossible to do this without adding further inputs (Reynolds numbers for the center and sidehulls) that should not have been necessary on strictly physical grounds. In addition, while Chaggar was able to obtain good predictions for $\mathrm{R}_{\mathrm{T}}$ and for the dimensionless Total Resistance Coefficient, it proved much harder in this work to predict Residual Resistance and the corresponding Residual Resistance Coefficient $\left(C_{R}\right)$. The latter of these functions was especially badly predicted, with no modification of the network architecture able to make a significant difference to the results.

It was then suspected the underlying difficulty was the relative scarcity of data; however, as will be seen later, the data are sufficient, but these resistance function approximation problems appear to be characterized by a very complex search space, requiring the deployment of an ANN learning method with strong stochastic search features, the Breeding PSO (BrPSO) algorithm used in this study and described in Section 4 below. It will also be demonstrated that BrPSO can solve the resistance prediction problems with only the three originally intended network inputs.

\section{PROBLEM DEFINITION AND DATA SET}

The Atlantic Center for the Innovative Design and Control of Small Ships (ACCeSS) is a consortium of university and industry partners. As a part of their current programme this center is looking into Tri-SWACH hydrodynamics and design, aiming for an improved understanding that will allow assessment of the overall performance of the vessel, providing confidence in its application. Although the Tri-SWACH hullform resembles that of a trimaran, its hydrodynamic characteristics are different and still not fully understood. Under the ACCeSS program a series of towing tank experiments, investigating the resistance characteristics of the Tri-SWACH design in calm water, have been conducted at the Stevens and Webb Institutes and at the United States Naval Academy (USNA).

In the ACCeSS towing tank experiments nine sidehull configurations were considered, comprised of three possible lateral positions of the sidehulls relative to the central hull (inboard (closest to central hull), middle, and outboard (furthest from central hull)) and three longitudinal (forward (furthest from stern), middle, and aft (closest to stern)) positions of the sidehulls relative to the centerhull strut front end (see Figure 1). These sidehull positions are illustrated in Figure 2.

However not all consortium members took measurements at all positions.Among the ACCeSS data, that from the Webb Institute [6] is the only complete set of experimental data from a single source; it was decided to follow [5] in using these data alone for resistance predictions, since experimental setups necessarily differ in detail, and [5] gives evidence of a discrepancy between resistance measurements for the same hull configurations obtained by different institutes.

The experimental setup used in the towing tank experiments of [6], from which our data were obtained, is shown in Figure 3.The sidehull locations considered are shown in outline in Figure 2, and relevant measurements given in Table 1 , in which \%LS is a dimensionless measure of longitudinal sidehull position (the longitudinal location from the centerhull strut front end divided by the length of the centerhull), and \% TS is a corresponding measure for the transverse sidehull position, given as the transverse location from the centerhull's center line divided by ten times the beam of the centerhull (the factor of 10 being a scale factor used in $[4,5]$ to bring $\% \mathrm{TS}$ into line with the magnitude of \%LS).

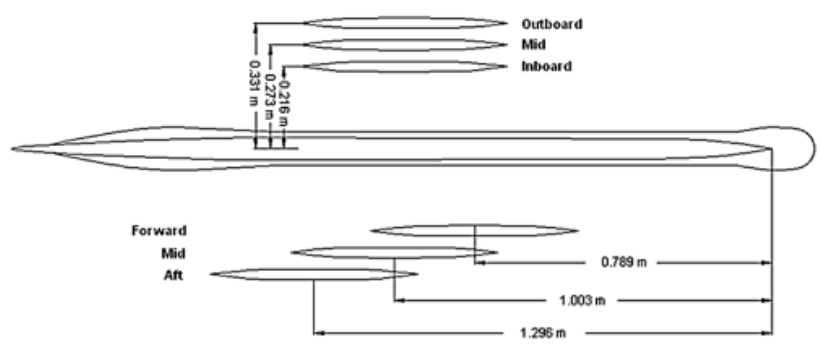

Figure 2. Tri-SWACH model sidehull locations for towing tank tests (figure from [3]).

Table 1. The nine sidehull positions considered, together with dimensionless descriptors of the geometry (used here as ANN inputs); highlighted column $\mathrm{E}$ will be the test data.

\begin{tabular}{|l|l|l|l|l|l|l|l|l|l|}
\hline position & A & B & C & D & E & F & G & H & I \\
\hline & $\begin{array}{l}\text { fwd- } \\
\text { outer }\end{array}$ & $\begin{array}{l}\text { fwd- } \\
\text { mid }\end{array}$ & $\begin{array}{l}\text { fwd- } \\
\text { inbd }\end{array}$ & $\begin{array}{l}\text { mid- } \\
\text { outer }\end{array}$ & $\begin{array}{l}\text { mid- } \\
\text { mid }\end{array}$ & $\begin{array}{l}\text { mid- } \\
\text { inbd }\end{array}$ & $\begin{array}{l}\text { aft- } \\
\text { outer }\end{array}$ & $\begin{array}{l}\text { aft- } \\
\text { mid }\end{array}$ & $\begin{array}{l}\text { aft- } \\
\text { inbd }\end{array}$ \\
\hline \% LS & 37 & 37 & 37 & 47 & 47 & 47 & 57 & 57 & 57 \\
\hline \% TS & 10.1 & 12.8 & 15.5 & 10.1 & 12.8 & 15.5 & 10.1 & 12.8 & 15.5 \\
\hline
\end{tabular}

Each combination of longitudinal and transverse sidehull positions contributes 34 data points, for Froude numbers between 0.1 and 0.5. Eight of the position combinations (columns A-D and F-I) will be used as training data, and column E (as also in [5]) as test data for the prediction of the resistance functions $\mathrm{R}_{\mathrm{T}}$ (Figure 4) and $C_{R}$ (Figure 5). It should however be noted that in contrast to $[4,5]$ predictions will here be based only on the minimal three inputs \% LS, \% TS, and Froude number $\left(\mathrm{F}_{\mathrm{r}}\right)$. 


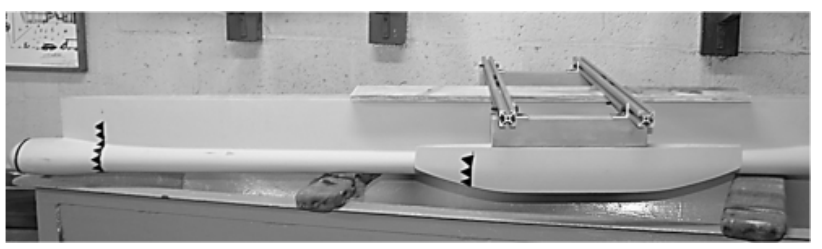

Figure 3. Tri-SWACH model used to obtain experimental towing tank resistance data (figure from [6]).

A validation data set, as will be discussed also in Section 5.1, was not used, as no evidence of overfitting was seen in preliminary experiments and the amount of non-testing data available was very limited. While it may seem surprising that overfitting was not observed, it is explicable by the fact that these are data gathered in strictly managed laboratory conditions, and that the standard procedure in towing tank experiments is to filter the raw signal data and average the measured resistance over the test time, thus reducing experimental noise to a minimum.

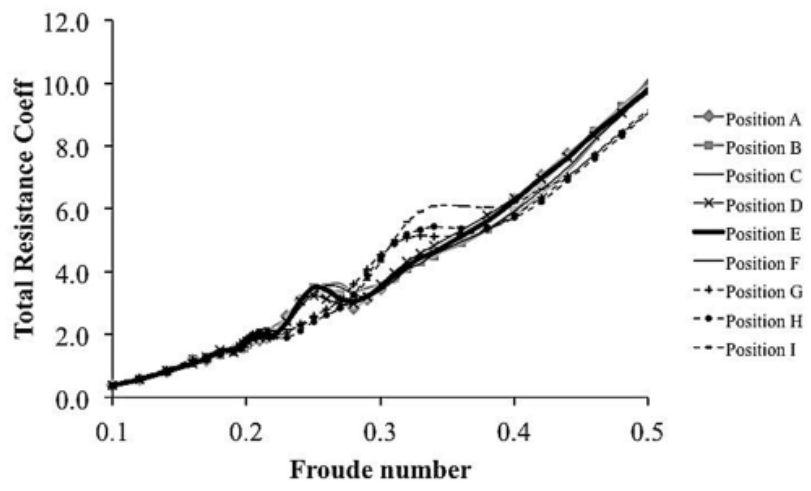

Figure 4. Total Resistance $\left(R_{T}\right)$ as a function of Froude number $\left(F_{r}\right)$; the curve in bold is the test data (mid-mid; sidehull position $E$ in Table 1).

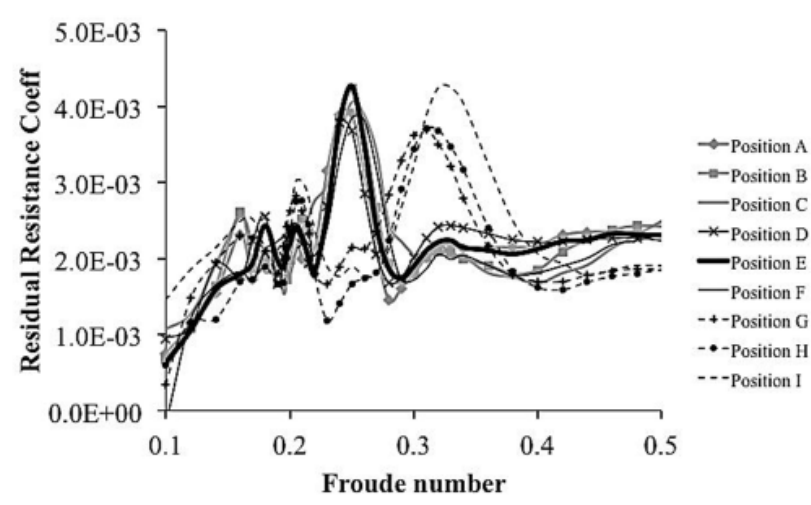

Figure 5. Residual Resistance Coefficient $\left(C_{R}\right)$ as a function of Froude number $\left(F_{r}\right)$; the curve in bold is the test data (midmid; sidehull position $E$ in Table 1), whose most significant features (the peak and side-lobes) could not be effectively predicted in [5] using any network architecture.

\section{METHODS}

\subsection{Particle Swarm Optimization (PSO)}

Particle swarm optimization (PSO) [7] is a biologically inspired, population based search algorithm that has been applied successfully in many areas for classification, prediction, and function approximation. The method is inspired by observations of social behavior in birds and other animals, and in its operation mixes the tendency of a swarm member (which here represents a suitably encoded solution to the problem at hand) both to return to positions (solutions) found previously to be effective and to follow a leader (that particle whose current position in the parameter space represents the best solution so far found by any particle). Solutions can be encoded in many ways, one of which is as the weights of a multilayer neural network.

In the algorithm introduced originally by Kennedy and Eberhart [7] every particle in a PSO swarm has a velocity $\mathbf{v}_{i}$ and position $\mathbf{x}_{i}$, where the latter will here correspond to the full list of weights possessed by the $i$ th (where $i=1 . . N$ ) particle, and the equations used to update each particle's velocity and position are

$$
\begin{gathered}
\mathbf{v}_{i, t+1}=W \mathbf{v}_{i, t}+\phi_{1} \beta_{1}\left(\mathbf{p}_{i, t}-\mathbf{x}_{i, t}\right)+\phi_{2} \beta_{2}\left(\mathbf{g}_{t}-\mathbf{x}_{i, t}\right) \\
\mathbf{x}_{i, t+1}=\mathbf{x}_{i, t}+\mathbf{v}_{i, t+1}
\end{gathered}
$$

where $\mathbf{p}_{i, t}$ is the personal best, the best position (weight set) found at time $t$ by particle (net) $i$, $\mathbf{g}_{t}$ (global best) is the best position found at this time by any particle, $\beta_{1}, \beta_{2}$ are random numbers chosen uniformly from the interval $[0,1]$, and $W$ is an iterationdecreasing inertia weight whose use improves swarm convergence.

This basic algorithm works well for many problems, but while it generally performs far better than gradient-based methods in search spaces with many local minima it can even so be troubled by convergence to sub-optimal solutions. For this reason it is frequently modified to a local-best form in which the role of the global best in (1a) is taken by a neighborhood best, a particle's neighborhood being defined for example in the ring topology as those particles indexed $i \pm 1$ in a list of $N$ particles numbered from 0 to $N-1$ (applying $\bmod N$ arithmetic operations), and the global best used as the final output solution being the best of these guiding local bests.

The PSO used here was initially of the ring topology form, but because of the complexity of the search space the algorithm was discovered insufficient for the Tri-SWACH resistance prediction problem. A novel and more powerful formalism was therefore deployed, Breeding PSO (BrPSO), that as well as the basic PSO search mechanisms described above also utilizes evolutionary elements.

\subsection{Breeding PSO (BrPSO)}

While standard PSO, as described above, is better able to navigate high-dimensional complex search spaces than a gradient-based method, its use of stochastic search is limited. In particular standard PSO, even in its local-best variant, lacks the ability to explore promising new areas far away from the directions of local and global convergence. BrPSO, a new form of PSO developed by one of us (SP) for use in this and other function approximation problems, attempts to resolve these issues by forming a hybrid between PSO, Differential Evolution [8,9], and Genetic Algorithms, using a PSO swarm that evolves rapidly via natural selection. 
Table 2. Comparison of performance of standard and Breeding PSO (BrPSO) on the $\mathbf{R}_{\mathrm{T}}$ training data set, in terms of Euclidean Distance (ED) and Mean Average Error (MAE) achieved after 10,000 iterations (50 runs)

\begin{tabular}{|c|c|c|c|c|c|}
\hline \multirow{2}{*}{$\begin{array}{l}\text { Training } \\
\text { Method }\end{array}$} & \multirow{2}{*}{ Architecture } & \multicolumn{2}{|c|}{ ED (training data) } & \multicolumn{2}{|c|}{ MAE (training data) } \\
\hline & & Average & Min & Average & Min \\
\hline Standard PSO & $3-20-1$ & $6.467 \pm 0.713$ & 4.878 & $0.245 \pm 0.016$ & 0.205 \\
\hline Standard PSO & $3-10-10-1$ & $3.066 \pm 0.386$ & 2.144 & $0.135 \pm 0.015$ & 0.100 \\
\hline BrPSO & $3-20-1$ & $3.613 \pm 1.024$ & 2.148 & $0.152 \pm 0.035$ & 0.094 \\
\hline BrPSO & $3-10-10-1$ & $2.110 \pm 0.446$ & 1.078 & $0.094 \pm 0.016$ & 0.051 \\
\hline
\end{tabular}

BrPSO is inspired by the CLPSO [10,11] and Global-Local Differential Evolution (GLDE) [12] algorithms. In CLPSO the information from two randomly selected competing local best particles (while for position updates only global best information is used). GLDE is in contrast based on the evolution/ crossbreeding of solutions within a fixed topology. BrPSO merges the successful components of both these algorithms with vanilla PSO.

The main difference between BrPSO and standard PSO is the breeding operation used in the former. On swarm initialization, each particle has a probability PPR assigned to it, from the range $[0,0.5]$; this will be the probability of its being potentially replaced by the child particle whose creation will next be described. In the mating procedure the global best 'mates' with the strongest particle out of two other randomly selected particles (picked with probabilities $1 /(N-1), 1 /(N-2))$, the strongest being that with the better personal best position. A child is then created in which the weight vector value associated with each dimension has a certain probability (cross-over probability PCO, here set to 0.5 ) of either taking on the equivalent value from the global best or from the personal best for the global best's selected partner. Once the child has been created its fitness is then compared to that of a chosen replacement candidate; if it is better this particle takes on the child's position, and that particle's personal best and the swarm global best are updated in the usual manner if applicable. In BrPSO there are thus two extra parameters, PPR and PCO, controlling the rate of additional exploration from breeding and from the formation of newly bred solutions respectively. If PPR is zero for all particles BrPSO reduces to vanilla PSO.

\subsection{Network Architectures and Data Processing}

In the experiments of [5] it was discovered better results could be obtained from nets with larger numbers of hidden units than had been used in the earlier work of [4]; it was thus decided here to focus on the use of nets with either a single layer of 20 hidden units (following [5], and referred to here-since three inputs will be used-as a 3-20-1 net) or two hidden layers of 10 hidden units each (3-10-10-1 net). The tanh function was used as a hidden layer neuron output function, functionally equivalent to the tansig used in [4, 5], and all swarms used in the experiments below consisted of 100 particles.

Input scaling: the definition of the \% TS parameter included a division by 10 to make it of similar magnitude to \%LS; it was decided here to divide both these inputs by an additional factor of 10 to make them of a similar magnitude to the Froude number input. Output scaling: none was done for the $\mathrm{R}_{\mathrm{T}}$ prediction problem, but given the very small magnitude of $C_{R}$ it was decided to multiply the targets by 100 during training to better separate the values. local-best PSO algorithm is adapted so that there is no fixed topology; a particle instead has a probability of obtaining

\section{RESULTS}

Different measures were used for training and for test performance assessment. It was discovered that training was most effective when using as fitness function the Euclidian Distance (ED) between the estimated and the target function values. However for the out-of-sample test data, the accuracy was measured in terms of the Mean Absolute Error (MAE), this choice being made in order to better compare our results against previous work [5] in which the MAE was the quoted measure.

As mentioned earlier in Section 3, the resistance prediction problems considered here, both of which used a data set derived solely from towing tank experiments in which the data gathered were postprocessed to remove noise, did not display any evidence of overfitting even after $10,000 \mathrm{~s}$ of iterations. This was discovered during initial runs in which randomly selected subsets $(20 \%$ of the 272 nontesting data points) were set aside for validation; no evidence of overfitting on the set-aside data was seen, only a degradation of training performance due to the lessened amount of available data. Thus it was decided to use all of the nontesting data in columns A-D and F-I of Table 1 for training.

\subsection{Prediction of Total Resistance $\left(R_{T}\right)$}

As Figure 3 shows, the variation of $R_{T}$ with Froude number $F_{r}$ for all sidehull configurations is in most places a smooth function, one that can in fact be well-approximated by a quadratic, $\mathrm{R}_{\mathrm{T}}\left(\mathrm{F}_{\mathrm{r}}\right)=25.269 \mathrm{~F}_{\mathrm{r}}^{2}+7.525 \mathrm{~F}_{\mathrm{r}}-0.7129$; it was thus decided to fit this function to the 272 training data examples and predict instead the residuals, notable only in the region of the prismatic humps.

The first series of experiments compared the performance of BrPSO with standard PSO on the training data set only, considering two alternate architectures with the same number of hidden units, but deployed differently in terms of layers. The objective of these initial experiments was to gain an understanding of the intrinsic level of difficulty of the problem and the degree to which BrPSO was able to outperform standard PSO using nets with the same number of hidden neurons, but deployed differently, in either one or two hidden layers. Because of the time-demanding nature of these experiments and the need to carry out a substantial number of runs it was decided to restrict the number of PSO iterations to 10,000 , and compare performance at that point.

The results of the above experiments are shown in Table 2 at the top of this page: it is clear BrPSO outperforms standard PSO for both architectures, and also that a net with two hidden layers is the more effective choice. The latter is an interesting observation in the light of the current high interest in deep learning architectures [13], and the many studies which have shown that deep nets are 
more effective than broad, shallow nets for problems with complex input data such as traffic sign recognition [14]. While deep neural network architectures have in the past been trained primarily using gradient-based methods such as error backpropagation there is now an interest in exploring non-gradient based learning for these networks, including nature-inspired methods such as PSO [15].

A series of 10 runs were then carried out using BrPSO and the 310-10-1 architecture, stopping when either no updates had been made to the swarm for 1000 iterations or after $10^{6}$ iterations, at which point the global best weights were used to make a prediction for the mid-mid test data. The average MAE was $0.118 \pm 0.026$, with $190867 \pm 97662$ iterations being taken (min 96061, max 419350), compared to a quoted average MAE of 0.132 in [5]. The predicted $R_{T}$ curve from an example run is shown in Figure 6. While these MAEs are not dissimilar it should again be noted that the BrPSO results are being obtained with only three inputs, \% TR, \%LS, and $\mathrm{F}_{\mathrm{r}}$, while the work of [4,5] required two additional Reynolds number inputs in order to make any reasonable prediction for the resistance functions.

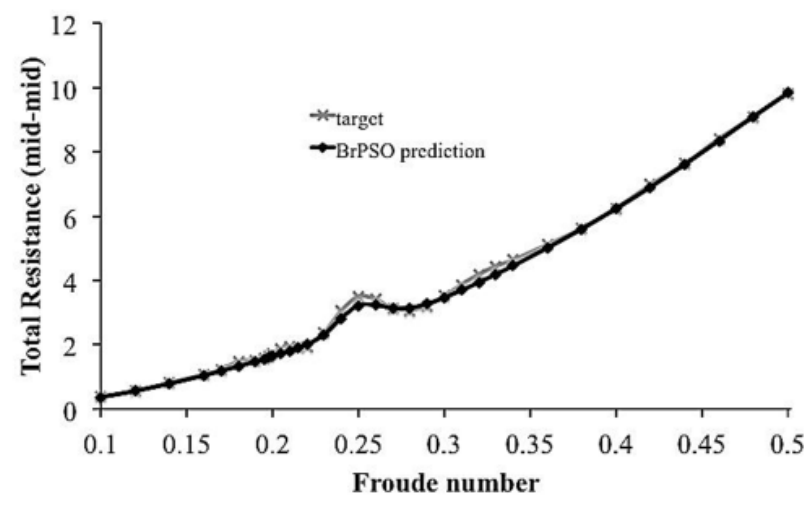

Figure 6. Test data comparison of BrPSO prediction with actual $\mathbf{R}_{\mathrm{T}}$

\subsection{Prediction of Residual Resistance Coefficient $\left(\mathbf{C}_{\mathbf{R}}\right)$}

As in the case of $R_{T}, 10$ runs were performed, with the same convergence criterion, giving in this case an average MAE of $2.424 \mathrm{e}-4 \pm 0.493 \mathrm{e}-4$, with $27656 \pm 11209$ iterations being taken ( $\mathrm{min}$ 15708, max 55332). When compared to the results of [5] (see Figure 7) it is clear BrPSO is performing far better for this function than Bayesian Regularization, which was in turn more effective than the Levenberg-Marquardt training used in the TriSWACH work of [4]. Using a challenging application in hydrology as a testbed it has been shown in [16] that Differential Evolution, which as discussed above is one of the key inspirations behind the BrPSO algorithm, is considerably more effective than Levenberg-Marquardt training, which was itself superior to a range of other nature-inspired methods including standard PSO.

An example $C_{R}$ prediction is shown in Figure 6, together with a representative example from [5] using training via Bayesian Regularization. While the BrPSO-generated curve does not quite hit the resistance peak around $F_{r}=0.25$, or show the full complexity of the sidelobes, it can be seen to capture the essential features of the function, and in particular the position of the 'hump'.

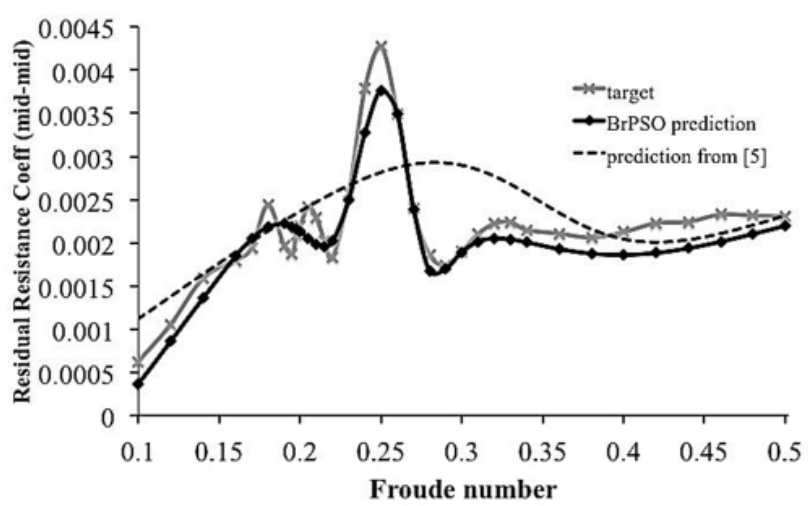

Figure 7. Test data comparison of BrPSO prediction with actual resistance coefficient $C_{R}$

\section{DISCUSSION}

This paper has introduced a new form of particle swarm optimization, Breeding PSO (BrPSO), and demonstrated its capacity to learn to approximate a complex function, the Residual Resistance Coefficient $C_{R}$, which is used to describe hull residual resistance in multihull ship design. The detailed approximation of this function was outside the capability of the gradient-based methods previously employed for this purpose [4,5] and also beyond the ability of standard PSO.

The curve showing Residual Resistance Coefficient as a function of Froude Number $\left(\mathrm{F}_{\mathrm{r}}\right)$ presented in Figure 7 confirms that BrPSO predictions match experimental data very closely. Although the peak value is not exactly captured, the position of the curve 'hump' is. The ability to correctly predict this range of Fr is of a significant value as it corresponds to a real life phenomenon: the superposition of the multihull wave patterns on the water surface between the hulls. While the value of the residual resistance will most likely change as the hullform design progresses, the position of the 'hump' will not change much and therefore brings confidence that appropriate configuration of centerhull and sidehulls is selected early on in Tri-SWACH design.

Although excellent resistance prediction was achieved by the BrPSO-trained ANN, the results obtained are limited by the experimental data to a one specific Tri-SWACH hullform, with only two parameters (\% TS and \%LS) varied in this configuration. However there are other significant sidehull parameters that impact resistance: length, breadth, volume, sidehull alignment against centerhull, and so on. It would be beneficial to expand the investigation for this configuration to consider a wider range of parameters, and to also consider variations in outrigger shapes. This will help us understand better the hydrodynamic behavior of these novel hullforms and the interaction between the centerhull and sidehulls.

As available experimental data is very limited, we might additionally explore the use of numerical simulations obtained by Computational Fluid Dynamics (CFD) tools. At the moment these simulations are still too resource expensive (in terms of time, hardware, and the availability of expert users) to be widely used in ship concept design investigation. Their application is usually limited to the later stages of design when the hullform type is already selected. Unfortunately at that point the main design 
parameters have already been fixed and any change would be very demanding (in terms of time and budget).

However CFD tools can also be used to generate systematic resistance data series that could be used for ANN training. This will allow us to cover not only a large range of Tri-SWACH parameters but also to expand the investigation to cover all trimaran type hullforms within one systematic data set.

If CFD is successfully coupled with ANN/BrPSO, these models would be able to quickly predict resistance for any configuration (within series limitations) and would therefore be very useful for design space exploration. Being in this way able to quickly predict resistance for various hullforms would allow a designer to quickly investigate a design space looking for optimal overall ship performance in the very early ship concept exploration phase and compare different hullform options: monohull, catamaran, trimaran, SWATH, Tri-SWACH and possibly more radical, innovative hullform configurations that are outside the scope of current design methodologies.

\section{ACKNOWLEDGMENTS}

ACCeSS is funded by the United States Navy's (USN) Office of Naval Research (ONR) under Grant Award No. N00014-10-10652. Thanks to this program, significant experimental data has been collated, forming a basis for this work.

\section{REFERENCES}

[1] Gougouldis, G. 2008. The utilization of artificial neural networks in marine applications: an overview. Naval Engineering Journal 120, 3 (Feb. 2008), 19-26.

[2] Couser, P., Mason, A., Mason, G., Smith, C, and Von Konsky, B. 2004. Artificial neural networks for hull resistance prediction. In Proc. 3rd International Conference on Computer Applications in the Marine Industries (Siguënza, Spain, May 09-12, 2004). COMPIT '04. Springer, Netherlands, 391-402.

[3] Royce, R., Mouravieff, A., and Zuzick. A. 2011. A trimaran resistance artificial neural network. In Proceedings of the 11th International Conference on Fast Ship Transportation (Honolulu, Hawaii, USA, Sept. 26-29, 2011). FAST '11. American Soc. Naval Engineers, Alexandria, VA, 717-726.

[4] Carter, A., Muk-Pavic, N., and McDonald,T. 2013. Resistance prediction using artificial neural networks for preliminary Tri-SWACH design. Trans. RINA XXX, A1, Intl. J. Maritime Engineering (June-Dec. 2013), 141-152.

[5] Chaggar, N. 2014. Tri-SWACH Resistance Prediction Using Artificial Neural Networks. MSc thesis, Dept. of Mechanical Engineering, University College London.
[6] Klag, J., and McMahon, I. 2007. Calm Water Resistance Study of a Novel Trimaran. Doctoral thesis.Webb Institute, Glen Cove, USA.

[7] Kennedy, J., and Eberhart, R. 1995. Particle swarm optimization. In IEEE International Conference Symposium on Neural Networks (Perth, Australia, Nov. 27-Dec. 01, 1995). IEEE Press, New York, NY, 1942-1948.

[8] Ilonen, J., Kamarainen, J.-K., and Lampinen, J. 2006. Differential evolution training algorithm for feed-forward neural networks. Neural Processing Letters 17, 1 (June 2006), 93-105.

[9] Storn, R. and Price, K. 1997. Differential evolution - a simple and efficient heuristic for global optimization over continuous spaces. Journal of Global Optimization 11, 4 (Dec. 1997), 341-359.

[10] Liang, J.J., Qin, A.K., and Baskar, S. 2006. Comprehensive learning particle swarm optimizer for global optimization of multimodal functions. IEEE Trans. Evolutionary Computation 10, 3 (June 2006), 281-295.

[11] Liang, J.J., Kai Qin, A., Suganthan, P.N., and Baskar, S. 2004. Evaluation of comprehensive learning particle swarm optimizer. In Proc. 11 th International Conference on Neural Information Processing (Calcutta, India, Nov. 22-25, 2004). ICONIP 2004. Springer-Verlag, Berlin Heidelberg, Germany, 230-235.

[12] Das, S., Abraham, A., Chakraborty, U., K., and Konar, A. 2009. Differential evolution using a neighborhood-based mutation operator. IEEE Trans. Evolutionary Computation 13, 3 (June 2009), 526-553

[13] Bengio, Y. 2009. Learning Deep Architectures for AI (Foundations and Trends in Machine Learning). Now Publishers Inc., Hanover, MA.

[14] Ciresan, D., Meier, U., Masci, J., and Schmidhuber, J. 2012. Multi-column deep neural network for traffic sign classification. Neural Networks, 32 (Feb. 2009), 333-338.

[15] Fortier, N., Sheppard, J.W., and Pillai, K.G. 2012. DOSI: Training artificial neural networks using overlapping swarm intelligence with local credit assignment. In Proc. 13th International Symposium on Advanced Intelligent Systems (Kobe, Japan, Nov. 20-24, 2012). ISIS '12. IEEE Press, New York, NY, 1420-1425.

[16] Piotrowski, A.P. and Napiorkowski, J.J. 2011. Optimizing neural networks for river flow forecasting - evolutionary computation methods versus the Levenberg-Marquardt approach. J. Hydrology, 407, 1 (Sept. 2011), 12-27. 